\title{
A Study of Clinical and Laboratory profile of Scrub Typhus in a Tertiary care Teaching Hospital
}

\author{
Atchuta Chytanya Paka ${ }^{1}$,Kamal Rajesh Jampana ${ }^{2}$ \\ ${ }^{1}$ Assistant Professor, Department of General Medicine, NRI Academy of Sciences, Mangalagiri Road, Chinakakani, Guntur, Andhra Pradesh- \\ $522503,{ }^{2}$ Associate Professor, Department of General MedicineNimra Institute of Medical Sciences, Nimra Nagar, Ibrahimpatnam, Jupudi, Vijayawada, \\ Andhra Pradesh-521456.
}

\section{Abstract}

Background: Scrub typhus, also known as bush typhus, is a disease caused by bacteria called Orientatsutsugamushi. This ricketssial infection is transmitted to humans through the bite of infected heptombidium mite larvae. Subjects and Methods: All patients were subjected to investigations to establish cause of febrile illness. After complete physical examination, routine laboratory investigations like CBC, serology for enteric fever, malaria, Scrub typhus and USG abdomen, chest x-ray, urine analysis and renal function tests were done in all patients. In all cases diagnosis was based on detection of antibodies using a single step rapid immunochromatography method. Results: 120 patients, who were admitted with undifferentiated acute febrile illness during the study period diagnosed to be suffering from Scrub typhus with positive antibodies, the following results were noted. Fever was the most common symptom seen in all the 120 patients (100\%). The duration of fever ranging from one to seven days was present in 94 patients (78.3\%), fever for 7-12days present in 5 patients(4.2\%) and fever for more than 2 weeks was present in 5 patients(4.12\%). Headache and vomitings were the commonly associated symptoms. Generalized muscle pain (myalgia) was present in 63patients(52.5\%). Headache was present in 64 patients $(53.3 \%)$, diarrohea was complained by 2 patients(1.7\%), vomiting in 47 patients(39.2\%), shortness of breath was present in 16 patients(13.3\%), abdominal pain was present in 17 patients(14.2\%) and skin rash was seen in 1 patient $(0.8 \%)$. Conclusion: Majority of the cases of Scrub typhus are seen in the cooler months of the year and in the rainy season. It has to be considered in the differential diagnosis of undifferentiated acute febrile illness.

Keywords: Clinical symptom \& Scrub typhus.

Corresponding Author: Dr. Kamal Rajesh Jampana,Associate Professor, Department of General Medicine, Nimra Institute of Medical Sciences, Nimra Nagar, Ibrahimpatnam, Jupudi, Vijayawada, Andhra Pradesh-521456.

Received: October 2019

Accepted: October 2019

\section{Introduction}

Scrub typhus, also known as bush typhus, is a disease caused by bacteria called Orientatsutsugamushi. ${ }^{[1]}$ This ricketssial infection is transmitted to humans through the bite of infected heptombidium mite larvae (chiggers). ${ }^{[2]}$ It predominantly occurs during the cooler months of the year as well as during the rainy season. ${ }^{[3]}$ Scrub typhus is an important cause of acute febrile illness and needs to be differentiated from other causes of febrile illness such as malaria, enteric fever, dengue fever, leptospirosisetc ${ }^{4}$.After the initial infection, the ricketssialinfection spreads systemically and the infected person develops various symptoms like fever, malaise, myalgia, rash, cough, lymphadenopathy and gastrointestinal disturbances. Various serological tests are available for diagnosis of Scrub typhus of which rapid immunochromatographic test is the most commonly used. The disease response to antibiotic is excellent. The aim of the present study is to present clinical manifestations, laboratory findings and treatment outcomes of adult Scrub typhus in a tertiary care setting.

\section{Subjects and Methods}

It is a prospective and retrospective study conducted from 2016-2018 at Nimra Institute of Medical Sciences, which is a tertiary care teaching hospital. All patients were subjected to investigations to establish cause of febrile illness. After complete physical examination, routine laboratory investigations like $\mathrm{CBC}$, serology for enteric fever, malaria, Scrub typhus and USG abdomen, chest $\mathrm{x}$-ray , urine analysis and renal function tests were done in all patients. In all cases diagnosis was based on detection of antibodies using a single step rapid immunochromatography method.

\section{Results}

Among 120 patients, who were admitted with undifferentiated acute febrile illness during the study period diagnosed to be suffering from Scrub typhus with positive antibodies, the following results were noted. The age of presentation in this study ranged from 18 years to 75 years. 75 patients(62.5\%) were males and 45 (38.3\%) were 
female.[Table.1].

Table 1: Age and Sex Distribution of Scrub Typhus.

\begin{tabular}{|l|c|c|c|}
\hline \multirow{2}{*}{$\begin{array}{c}\text { Age Group } \\
\text { (In Years) }\end{array}$} & \multicolumn{2}{|c|}{ Gender } & \multirow{2}{*}{ No. of Cases } \\
\cline { 2 - 3 } & Male & Female & \\
\hline 15-20yrs & 6 & 4 & $10(8.3 \%)$ \\
\hline $21-30 y r s$ & 22 & 11 & $32(26.7 \%)$ \\
\hline Above 30yrs & 47 & 31 & $78(65.0 \%$ \\
\hline Total no. of Patients & $\mathbf{7 5 ( 6 2 . 5 \% )}$ & $\mathbf{4 5 ( 3 8 . 3 \% )}$ & $\mathbf{1 2 0}$ \\
\hline
\end{tabular}

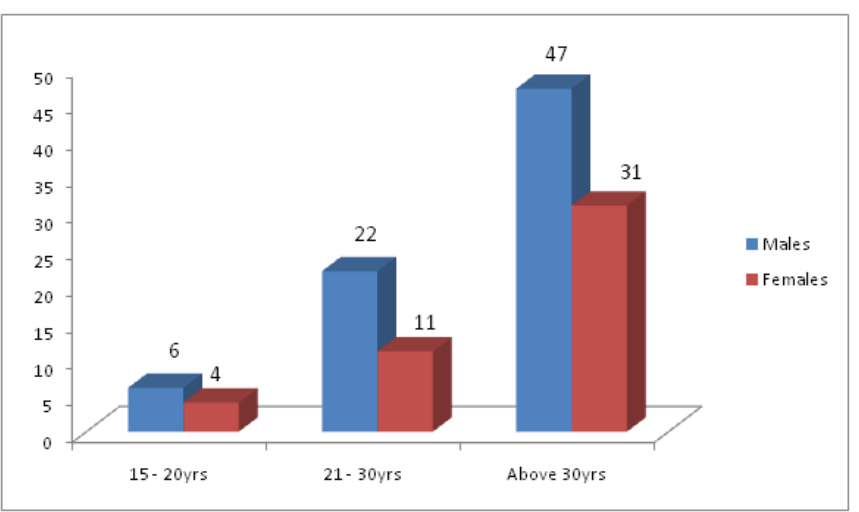

Chart 1: Age and Sex Distribution of Scrub Typhus

Table 2: Clinical Features of Scrub Typhus at Presentation.

\begin{tabular}{|l|c|c|}
\hline \multicolumn{1}{|c|}{ Clinical Feature } & No. of Patients & \\
\hline Fever & 120 & 1 \\
\hline Headache & 64 & 53 \\
\hline Myalgias & 63 & 5 \\
\hline Vomitings & 47 & 39 \\
\hline Cough & 28 & 23 \\
\hline Abdominal pain & 17 & 1 \\
\hline Shortness of breath & 16 & 1 \\
\hline Eschar & 9 & 7 \\
\hline Diarrhoea & 2 & 1 \\
\hline Skin rash & 1 & 0.48 \\
\hline
\end{tabular}

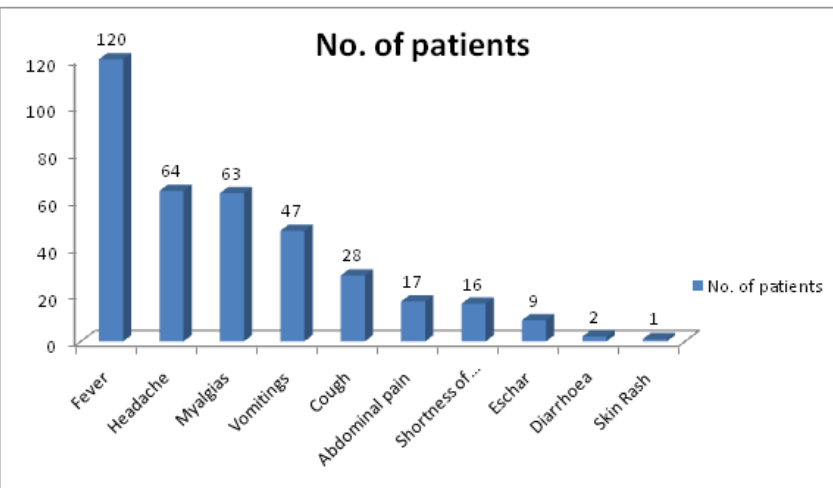

Chart 2: Clinical Features of Scrub Typhus at Presentation.

Table 3: Complications of Scrub Typhus.

\begin{tabular}{|l|c|c|}
\hline \multicolumn{1}{|c|}{ Complications } & No. of Patients & Percentage \\
\hline Thrombocytopenia & 37 & $30.8 \%$ \\
\hline Hepatitis & 27 & $22.5 \%$ \\
\hline AKI & 7 & $5.8 \%$ \\
\hline MODS & 5 & $4.2 \%$ \\
\hline ARDS & 4 & $3.3 \%$ \\
\hline Encephalopathy & 4 & $3.3 \%$ \\
\hline
\end{tabular}

No. of Patients

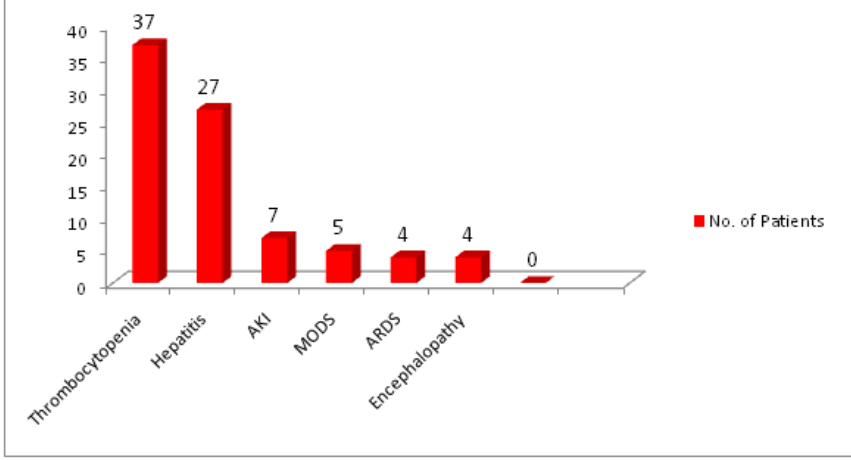

Chart 3: Complications of Scrub Typhus.

\section{Discussion}

Fever was the most common symptom seen in all the 120 patients $(100 \%)$. The duration of fever ranging from one to seven days present in 94 patients (78.3\%), fever for 7-12days present in 5 patients $(4.2 \%)$ and fever for more than 2 weeks present in 5 patients $(4.12 \%)$. Headache and vomiting were the commonly associated symptoms. Generalized muscle pain (myalgia) was present in 63patients $(52.5 \%)$. Headache waPpresentrindrat patients $(53.3 \%)$, diarrohea was complained $100 \% 2$ patients(1.7\%), vomiting in 47 patients(39.2\%), 53 stiortness of breath was present in 16 patients(13.3\%), $52.5 \%$ \%ominal pain was present in 17 patients $(14.2 \%)$ and skin 39 1 $16 \%$ was seen in 1 patient $(0.8 \%)$ (Table 2$)$. In 9 patients 14.16\% an Eschar was detected after careful examination in 13.3arious sites like left axilla, hypochondriac region, left groin, $7.5 \times x m s$, gluteal region, left abdomen and neck. Prominent 1. Treamatological abnormalities included thrombocytopenia 0.98 pesent in 37patients( $30.8 \%)$ and elevated levels of bilirubin, transaminases were also seen in 27 patients(22.5\%). A CNS manifestation with features of meningoencephalitis was present in 4 patients $(3.3 \%)$. All the patients were started on either oral or intravenous doxycycline along with supportive care.

Scrub typhus is a noticeable mite borne disease with its incidence being highest in Korea. There have so far been a few reports on severe complications and the mortality rate of Scrub typhus ${ }^{5}$. Disease occurrence is more in rainy season and occurs in persons who engage in occupational or recreational behaviour that brings then in contact with mite infested habitats such as brush and grass.

Scrub typhus can be a mild, moderate or severe febrile illness with non-specific clinical features. Fever was the most common symptom reported by all patients in our study.

Eschar at the site of attachment of the larval mite (chigger) is considered highly suggestive of Scrub typhus but occurs in a variable proportion of patients in different studies $^{6}$. In our study, eschar was present in 9 patients (7.5\%) and the most common site was left groin and neck region. Previous studies from India have reported meningoencephalitis in $9.5-23 \%$ of patients ${ }^{[7]}$ in our study meningoencephalitis was present in 4 patients. 


\section{Haspital}

\section{Conclusion}

In conclusion, the majority of the cases of Scrub typhus are seen in the cooler months of the year and in the rainy season. It has to be considered in the differential diagnosis of undifferentiated acute febrile illness. In view of the increasing burden on public health system, there should be a high index of suspicion in patients presenting with acute febrile illness. Early diagnosis and prompt intervention may help in reducing the morbidity and mortality associated with Scrub typhus infection.

\section{References}

1. Https://www.cdc.gov/typhus/scrub/index.html

2. https://www.iamat.org > risks > scrub typhus

3. https://www.ijpediatrics.com. Radhakumar, PurusothamanSrinivasan. A study of clinical and laboratory profile of scrub typhus in children in a tertiary hospital in south India.

4. www.ijph.inAroma Oberoi, Shereen Rachel Varghese.Scrub typhus- an emerging entity: A study from a tertiary care hospital in North India.

5. Varghese GM, TrocobridgeP, JanardhananJ, Thomas K, Peter JV, Mathews P, Abraham OC, JavithaML. Scrub typhus among hospitalized patients with febrile illness in South India

6. ChogleAR. Diagnosis and treatment of Scrub typhus The Indian scenario. $\mathrm{j}$ assoc physicians India 2010:58:11:12

7. Vivekanandan M. Mani A, Priya YS, Singh AP, Jyakumar S, Purty S. Outbreak of Scrub typhus in Pondicherry. Jassoc physicians India 2010:58:24-8

Copyright: () the author(s), 2019. It is an open-access article distributed under the terms of the Creative Commons Attribution License (CC BY 4.0), which permits authors to retain ownership of the copyright for their content, and allow anyone to download, reuse, reprint, modify, distribute and/or copy the content as long as the original authors and source are cited.

How to cite this article: Paka AC, Jampana KR. A Study of Clinical and Laboratory profile of Scrub Typhus in a Tertiary Care Teaching Hospital. Acad. J Med. 2019;2(2):21-23.

DOI: dx.doi.org/10.21276/ajm.2019.2.2.7

Source of Support: Nil, Conflict of Interest: None declared. 